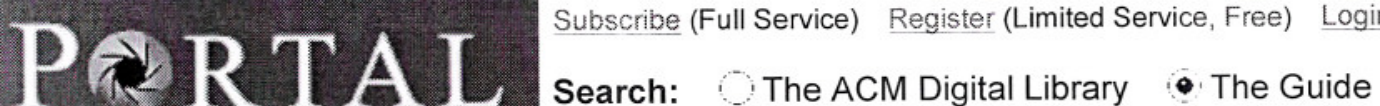

THE GUIDE TO COMPUTING LITERATURE

Call Admission Control in Cellular Mobile Networks: A Learning Automata Approach

Source Lecture Notes In Computer Science; Vol. 2510 archive

Proceedings of the First EurAsian Conference on Information and Communication Technology table of contents Pages: 450 - 457

Year of Publication: 2002

ISBN:3-540-00028-3

Authors

Hamid Beigy

Mohammad Reza Meybodi

Publisher

Springer-Verlag London, UK

Tools and Actions:

Discussions Find similar Articles Review this Article

Save this Article to a Binder Display Formats: BibTex EndNote ACM Ref

$\uparrow$ Collaborative Colleagues:

Hamid Beigy:

M. R. Meybodi

Mohammad Reza Meybodi

Mohammad Reza Meybodi: Hamid Beigy

Mohammad Mehdi Homayounpour

Jahanshah Kabudian

Farhad Oroumchian

Masoud Tashakori

The ACM Portal is published by the Association for Computing Machinery. Copyright $\odot 2006$ ACM, Inc.

Terms of Usage Privacy Policy Code of Ethics Contact Us

Useful downloads: Adobe Acrobat $_{\text {QuickTime Ai Windows Media Player }} \mathrm{S}_{\text {Real Player }}$ 


\section{Call Admission Control in Cellular Mobile} Networks: A Learning Automata Approach

Hamid Beigy and Mohammadreza Meybodi

\author{
Soft Computing Laboratory \\ Computer Engineering Department \\ Amirkabir University of Technology \\ Tehran, Iran
}

$\{$ beigy, meybodi\}@ce.aku.ac.ir

called guard channels, for handoff calls (say $C-T$ channels). Whenever the channel occupancy exceeds the certain threshold $T$, the GC policy rejects new calls until the channel occupancy goes below $T$. The GC policy accepts handoff calls until the channel occupancy goes below $T$. The calls as long as channels are available. It has been shown that there is an optimal
cals a threshold $T^{*}$ in which $B_{n}$ is minimized subject to the hard constraint on $B_{h}$ [3]. An algorithm for finding such optimal threshold is given in [3]. In order to have more control on $B_{h}$ and $B_{n}$, limited fractional guard channel policy (LFG) is more control on $B_{h}$ and $B_{n}$, limited fractional guard channel policy (LFG) is
introduced [1]. The LFG can be obtained from FG policy by setting $\pi_{k}=1$, $0<k<T, \pi_{T}=\pi$, and $\pi_{k}=0, T<k<C$. It has been shown that there are antimal threshold $T^{*}$ and an optimal value of $\pi^{*}$ in which $B_{n}$ is minimized subject to the hard constraint on $B_{h}[1]$. The algorithm for finding such optimal parameters is given in [1]. In [4], a restricted version of FG policy, called uniform fractional guard channel policy (UFG), is introduced. The UFG policy accepts new calls with probability of $\pi$ independent of channel occupancy. The UFG can be obtained from FG by setting $\pi_{k}=\pi, 0 \leq k<C$. In order to find the optimal value of parameter $\pi$, in [4] a binary search algorithm is given.

In this paper a reinforcement learning based algorithm is given to find the optimal value of $\pi$ for the UFG policy. In context of CAC, the use of reinforcement learning techniques can lead to good solution in reasonable time. Instead of relying on a known teacher, the system is designed to learn an optimal assignment policy by directly interacting with the environment. Learning automaton (LA) is a reinforcement learning technique and has been used successfully in many applications such as telephone and data network routing $[5,6]$, solving NP-Complete problems $[7,8,9]$ and capacity assignment $[10]$, to mention a few. In this paper, we propose a LA based CAC algorithm for cellular networks. In this algorithm, LA is used to determine acceptance/rejection probability $(\pi)$ of new calls.

The rest of this paper is organized as follows: Section 2 presents performance parameters of UFG policy and gives an algorithm to find the optimal value of parameter of UFG policy. The LA is given in section 3 and the proposed CAC policy is given in section 4 . The computer simulations is given in section 5 and section 6 concludes the paper. increases the expected rate of handovers per call. As a consequence, some network performance parameters such as blocking probability of new calls $\left(B_{n}\right)$ and dropping probability of handoff calls $\left(B_{h}\right)$ are affected. In order to maintain $B_{h}$ and $B_{n}$ at a reasonable level, call admission control (CAC) algorithms are used, which play a very important role in the cellular networks because directly control $B_{n}$ and $B_{h}$. Since $B_{h}$ is more important than $B_{n}$. CAC policies give the higher priority to handoff calls. This priority is implemented through allocation of more resources (chamnels) to handoff calls. A general CAC policy, called fractional guard channel policy (FG), accepts new calls with a probability that depends on the current channel occupancy and accepts the handoff calls as long as channels are available [1]. Suppose that the given cell has $C$ full duplex channels. The FG policy uses a vector $\Pi=\left\{\pi_{0}, \ldots, \pi_{C-1}\right\}$ to accept the new calls, where $0 \leq \pi_{i} \leq 1$ and $0 \leq i<C$. The $\mathrm{FG}$ policy accepts new calls with probability of $\pi_{k}$ when $k(0 \leq k<C)$ channels are busy. Unfortunately, there is no algorithm to find the optimal vector $\Pi^{*}$. A restricted version of FG is called guard channel policy (GC) [2]. The GC policy reserves a subset of channels,

\section{The Uniform Fractional Guard Channel Policy}

In UFG policy, handoff calls are accepted as long as channels are available and new calls are accepted with probability $\pi$, which is independent of channel occupancy. The description of UFG policy is given algorithmically in figure 1 . In the next subsections, we first study the blocking performance of UFG and then give a binary search algorithm to find the optimal value of parameter $\pi$.

\subsection{The Blocking Performance of UFG}

In what follows, we study the blocking performance of UFG policy. The blocking performance of UFG policy is computed based on the following assumptions. 


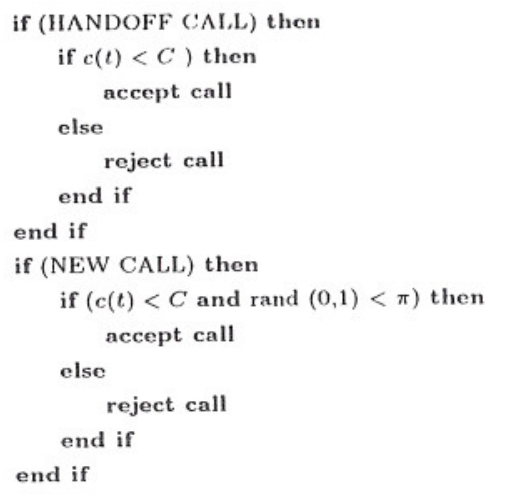

Fig. 1. Unform fractional guard channel policy

1. The arrival process of new and handoff calls is poisson process with rate $\lambda_{n}$ and $\lambda_{h}$, respectively.

2. The holding time for both types of calls are exponentially distributed with mean $\mu^{-1}$.

3. The time interval between two calls from a mobile host is much greater than the mean call holding time.

4. Only mobile to fixed calls are considered.

5. The network is homogenous.

The above first three assumptions have been found to be reasonable as long as the number of mobile hosts in a cell is much greater than the number of channels allocated to that cell. The fourth assumption makes our analysis easier and the fifth one lets us to examine the performance of a single network cell in isolation.

Suppose that the given cell has a limited number of full duplex chamel: $C$, in its channel pool. We define the state of a particular cell at time $t$ to be the number of busy channels in that cell and is represented by $c(t)$. The $\{c(t) \mid t \geq 0\}$ is a continuous-time Markov chain (birth-death process) with states $0,1, \ldots, C$. The state transition rate diagram of a cell with $C$ full duplex channels and UFG call admission policy is shown in figure 2 .
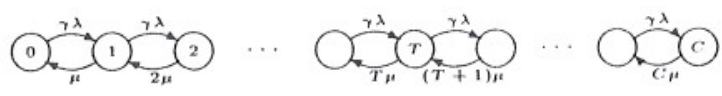

Fig. 2. Markov chain model of cell

At the state $0 \leq n<C$, new calls are accepted with probability $0 \leq \pi<1$ and handoff calls are accepted with probability 1 . Both types of calls are blocked in state $C$. Thus, the state dependent arrival rate in the birth-death process is equal to $[a+(1-a) \pi] \lambda$, where $a=\frac{\lambda_{h}}{h}$ is the handoff traffic in Erlangs and $\lambda=\lambda_{h}+\lambda_{n}$ is total traffic seen by the cell. Because of the structure of the Markov chain, we can easily write down the steady-state balance equations. Define the steady state probability $P_{n}=\lim _{t \rightarrow \infty} \operatorname{Prob}[c(t)=n]$ for state $n=0,1, \ldots, C$. Then, the following expression can be derived for $P_{n}(n=0,1, \ldots, C)$.

$$
P_{n}=\frac{(\rho \gamma)^{n}}{n !} P_{0}
$$

where $\gamma=[a+(1-a) \pi]$ and $\rho=\lambda / \mu$ is the total traffic in Erlangs as seen by a cell and $P_{0}$ is the probability that all channels are free. The value of $P_{0}$ is calculated by the following expression.

$$
P_{0}=\left[\sum_{n=0}^{C} \frac{(\rho \gamma)^{n}}{n !}\right]^{-1}
$$

Thus, the dropping probability of handoff calls, $B_{h}(C, \pi)$, id given by

$$
B_{h}(C, \pi)=\frac{(\rho \gamma)^{C}}{C !} P_{0}
$$

Similarly, the blocking probability of new calls, $B_{n}(C, \pi)$ is equal to

$$
\begin{aligned}
B_{n}(C, \pi) & =\sum_{n=0}^{C-1}(1-\pi) P_{n}+P_{C} \\
& =1-\pi\left[1-B_{h}(C, \pi)\right]
\end{aligned}
$$

\subsection{Finding of Optimal Parameter of UFG Policy}

The objective of our call admission control policy is to find a $\pi^{*}$ that minimizes $B_{n}(C, \pi)$ with the constraint that $B_{h}(C, \pi)$ must be at most $p_{h}$. The value of $p_{h}$ specifies the QoS of the network. It is too complex to obtain an exact solution for this problem. Hence, a search algorithm is given to determine the optimal value of $\pi$ for given traffic and constraint $p_{h}$. This algorithm is given in figure 3

Since the proposed algorithm needs all parameters of input traffic, which are unknown and possibly time varying, we introduce a LA based algorithm for determination of parameter $\pi$.

\section{Learning Automata}

The automata approach to learning involves the determination of an optimal action from a set of allowable actions. An automaton can be regarded as an abstract object that has finite number of actions. It selects an action from its finite set of actions. This action is applied to a random environment. The random enviromment evaluates the applied action and gives a grade to the selected 


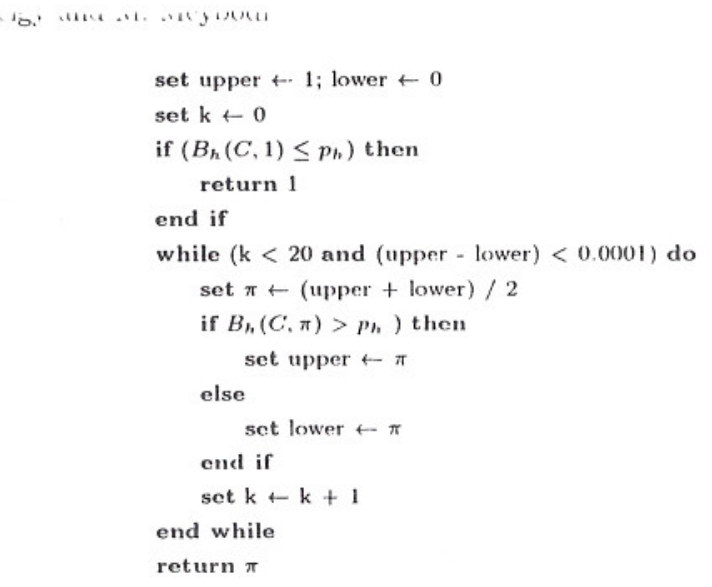

Fig. 3. Algorithm for determination of $\pi^{*}$

action of automata. The response from enviromment (i.e. grade of action) is used by automata to select its next action. By continuing this process, the automaton learns to select an action with best grade. The learning algorithm used by automata to determine the selection of next action from the response of environment. An automaton acting in an unknown random environment and improves its performance in some specified manner, is referred to as learning automaton LA can be classified into two main fanilies: fixed structure learning automata and variable structure learning automata [11]. Variable structure learning automata is represented by triple $\langle\beta, \alpha, T\rangle$, where $\beta$ is a set of inputs actions, $\alpha$ is a set of actions, and $T$ is learning algorithm. The learning algorithm is a recurrence relation and is used to modify the state probability vector. It is evident that the crucial factor affecting the performance of the variable structure learning automata, is learning algorithm for updating the action probabilities. Various learning algorithms have been reported in the literature. Let $\alpha_{i}$ be the action chosen at time $k$ as a sample realization from probability distribution $p(k)$. In linear reward- $\epsilon$ penalty algorithm $\left(L_{R-\epsilon P}\right)$ scheme the recurrence equation for updating $p$ is defined as

$$
\begin{gathered}
p_{j}(k+1)=\left\{\begin{array}{l}
p_{j}(k)+a \times\left[1-p_{j}(k)\right] \text { if } i=j \\
p_{j}(k)-a \times p_{j}(k) \quad \text { if } \quad i \neq j
\end{array} \text { if } \beta(k)=0\right. \\
p_{j}(k+1)=\left\{\begin{array}{ll}
p_{j}(k) \times(1-b) \quad \text { if } i=j \\
\frac{b}{r-1}+p_{j}(k)(1-b) \text { if } \quad i \neq j
\end{array} \text { if } \beta(k)=1\right.
\end{gathered}
$$

The parameters $0<a<1$ and $0<b \ll a$ represent step length.s and $r$ is the number of actions for LA. The $a$ and $b$ determine the amount of increase and decreases of the action probabilities, respectively. If the $a$ equals to $b$ the recurrence equations (5) and (6) is called linear reward penalty $\left(L_{R} \ldots p\right)$ algorithm.

\section{LA Based CAC Strategy}

In this section, we introduce a LA based call admission algorithm (figure 4). This algorithm is used to determine admission probability $\pi$ when parameters $a$ and $\rho$ (or equivalently $\lambda_{h}, \lambda_{n}$ and $\mu$ ) are unknown or probably time varying. The proposed CAC strategy adjusts parameter $\pi$ as long as network operates. This algorithm gives a higher probability to handoff calls by allowing handoff calls to be accepted with higher probability than new calls. This algorithm can be described as follows: The proposed algorithm uses one reward-penalty type LA with two actions in each cell. The action set of this automaton corresponds to $\{$ ACCEE'T',RE.JEC'I' $\}$. The automaton associated to each cell determines the probability of acceptance of new calls $(\pi)$. Since initially the values of $a$ and $\rho$ are unknown, the probability of selecting these actions are set to 0.5 . When a handoff call arrives, it is accepted as long as there is a free channel. If there is no free channel, the handoff call is blocked. When a new call arrives to a particular cell LA associated to that cell chooses one of its actions. Let $\pi$ be the probability of selecting the action ACCEPT. Thus, the LA accepts new calls with probability $\pi$ as long as there is a free channel and rejects them with probability $1-\pi$. If action ACCEPT is selected by automaton and the cell has a free channel, then action ACCEPT is rewarded. If there is no free chamel to be allocated to the arrived new call, the call is blocked and action ACCEPT is penalized. When the automaton selects action REJECT, it estimates the dropping probability of handoff calls $\left(\hat{B}_{h}\right)$. If the current estimate of dropping probability of handoff calls is less than the given threshold $p_{h}$ and there is a free channel, then the new call is accepted and action RE.JECT is penalized. This rule causes that the channels used more efficiently. In other case, the new call is rejected and action REJECT is rewarded.

\section{Simulation Results}

In this section, we compare performance of the uniform fractional guard channel and the LA based call admission policies. The results of simulations are summarized in table 1. The simulation is based on the single cell of homogenous cellular network system. In such network, each cell has 8 full duplex channels $(C=8)$. In the simulations, new call arrival rate is fixed to 30 calls per minute $\left(\lambda_{n}=30\right)$, chanuel holding time is set to 6 seconds $\left(\mu^{-1}=6\right)$, and the handoff call traffic is varied between 2 calls per minute to 20 calls per minute. The results listed in table 1 are obtained by averaging 10 runs from $2,000,000$ seconds simulation of each algorithm. The objective is to minimize the blocking probability of new calls subject to the constraint that the dropping probability of handoff calls is less than 0.01 . The optimal parameter of uniform fractional guard channel policy is obtained by algorithm 3 .

By carefully inspecting the table 1, it is evident that for some range of input traffics, the performance of the proposed policy is close to the performance of the uniform fractional guard chamel policy. 


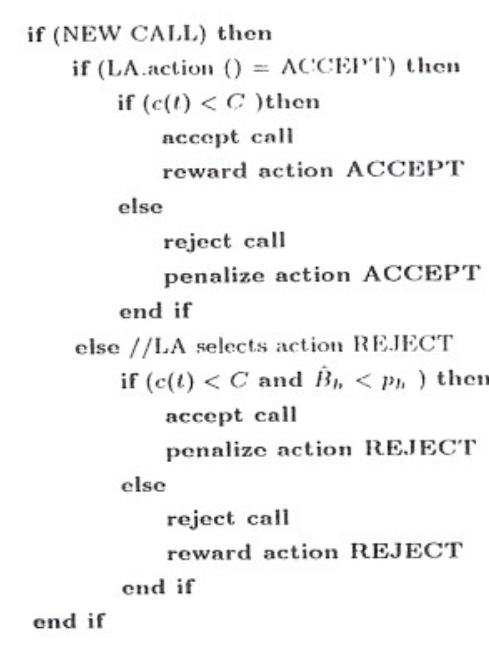

Fig. 4. LA based call admission control algorithm!

Table 1. Minimize $B_{n}$ such that $B_{h} \leq 0.01$

\begin{tabular}{c|c|cc|cc}
\multirow{2}{*}{ Case } & \multirow{2}{*}{$\lambda_{h}$} & $\begin{array}{c}\text { Uniform Fractional Guard } \\
\text { Channel Policy }\end{array}$ & \multicolumn{2}{|c}{$\begin{array}{c}\text { Based Uniform Fractional } \\
\text { Guard Channel Policy }\end{array}$} \\
\cline { 3 - 6 } & $B_{n}$ & $B_{h}$ & $B_{n}$ & $B_{h}$ \\
\hline 1 & 2 & 0.023935 & 0.024675 & 0.054314 & 0.023388 \\
2 & 4 & 0.089897 & 0.023639 & 0.088707 & 0.025217 \\
3 & 6 & 0.15725 & 0.022202 & 0.147586 & 0.024847 \\
4 & 8 & 0.223872 & 0.020367 & 0.193917 & 0.024625 \\
5 & 10 & 0.289849 & 0.019248 & 0.193917 & 0.024625 \\
6 & 12 & 0.356866 & 0.017607 & 0.249867 & 0.024294 \\
7 & 14 & 0.424072 & 0.016390 & 0.289657 & 0.025141 \\
8 & 16 & 0.489967 & 0.015076 & 0.391456 & 0.024460 \\
9 & 18 & 0.557026 & 0.013939 & 0.444263 & 0.023861 \\
10 & 20 & 0.623746 & 0.013318 & 0.488290 & 0.024800
\end{tabular}

\section{Conclusions}

In this paper, we introduced a LA based call admission control policy for cellular networks. In the proposed policy, a LA is used to accept/reject new calls. This call admission policy can be considered as adaptive miform fractional guard channel policy. In order to study the performance of the proposed call admission policy, the computer simulations are conducted. The simmlation results show that for some range of input traffics, the performance of the proposed approach is close to the performance of the uniform fractional guard channcl policy. Since LA are adaptive and don't need any information about its enviromment, the proposed policy is fully adaptive and doesn't recuire any information aloont the input traftics.

\section{References}

1. R. Raunjee, D. Towsley, and R. Nagarajan, "On Optimal Call Admission Control in Cellular Networks," Wireless Networks, vol. 3, pp. 29-41, 1997

D. Whaflic Modelling and Performance Analysis for CelluDobile Radio Telephone Systems with Priotrized and Nonpriotorized Handoffs Procedure," IEEE Transactions on Vehicular Technology, vol. 35, pp. 77-92, Aug. 1986.

3. G. Haring, R. Marie, R. Puigjaner, and K. Trivedi, "Loss Formulas and Their ApG. Thation to Cellular Networks," IEEE Transactions on Vehicular Technology, vol. 50, pp. 664-673, May 2001.

Theybodi, "Uniform Fractional Guard Channel," in Proceed 4. Heigy and M. R. Meybodi, "Uniform Fractional Guard Channel, in ProcedOrlando, USA, July 2002

5. P. R. Srikantakumar and K. S. Narendra, " $\Lambda$ Learning Model for Routing in Telephone Networks," SIAM Journal of Control and Optimization, vol. 20, pp. 34-57, Jan. 1982.

G. V Nedzelnitsky and K. S. Narendra, "Nonstationary Morlels of Learning Au. O. Nedzelnitsky and K. S. Narchition Networks," IEEE Transactions on Systomata Routing in Data Communication Networks, 1015, Nov. 1987.

tems, Man, and Cybernetics, vol. SNC-17, pp). 1004-1015, Nov. 1987 . . B. J. Oommen and 1996.

8. H. Beybodi, "An Algorithm Based on Learning Automata for Det Units in Three Layers Neural Networks," Amirkabir Journal of Science and Technology, vol. 12, no. 46, pp. 111-136, works,

M. R. Meybodi and H. Beigy, "Neural Network Engineering Using Learning Autom works," Journal of Faculty of Engineering, vol. 34, pp. 1-2f "' y 2001

10. B. J. Oommen and T. D. Roberts, "Continuous Learning Automata Solutis to B. Capacity Assignment Problem," IEEE Transactions on Commputers, vol. 49, the Capacity Assignment

pp. 608-620, June 2000 . Thathachar, Learning Automata: An Introduction. New York: Printice-Hall, 1989. 


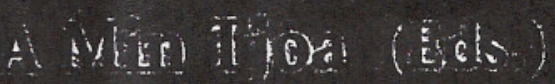

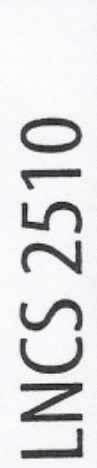

\section{First EurAsian Conference} Shiraz, Iran, October 2002 Proceedings

\section{eur@sia}

iCT 2002 